\title{
Efficient Broadcast Authentication using TSG Algorithm for WSN
}

\author{
M. Premkumar \\ Assistant Professor/ECE \\ Sri Subramanya College of \\ Engineering and Technology \\ Palani, Tamil Nadu, India
}

\author{
M. Kathiravan \\ Assistant Professor/ECE \\ Dr. Nallini Institute of \\ Engineering and Technology \\ Dharapuram, Tamil Nadu, India
}

\author{
R. Thirukkumaran \\ M.E.-communication systems \\ Karpagam College of \\ Engineering \\ Coimbatore, Tamil Nadu, India
}

\begin{abstract}
Wireless Sensor Networks (WSN) is an emerging technology that has a wide range of applications including environment monitoring, medical systems, robotic exploration and smart places. In most of the sensor network sink node send broadcast information to all other sensor nodes. Broadcast authentication is an important security mechanism in a Wireless Sensor Network. . Base station broadcast the public key to all the sensor nodes. Broadcast message authenticated by using public key. If single key is used throughout the life time, adversaries can easily listen to the communication between the sensor nodes and the base station. To overcome this short term keys are introduced. In shortPK, the base stations broadcast a set of many short length encrypted keys for all the sensor nodes for every short term. The encrypted key contains message and next phase key. Due to long propagation of encrypted keys throughout the network causes propagation delay, decryption failure and packet loss. To overcome this problem we proposed Tree based Short term Group key Algorithm (TSGA) to reduce packet loss and enhance security mechanism by using short term group key in wireless sensor networks. The basic idea of the project is to use many short length group keys to provide the broadcast authentication in wireless sensor networks. The overall network is constructed as tree from base station and the parent and child nodes are established for different levels. Depends on level in tree network group will be formed. The encryption and decryption takes place within the group itself so the propagation delay of public key and packet loss will be reduced. Our simulation result shows that the packet loss is reduced and the data transmission also more secured than previous schemes..
\end{abstract}

\section{General Terms}

Wireless sensor networks, security, broadcast, authentication, ECC.

\section{Keywords}

Depth creation, group key establishment, parent node, TSGA

\section{INTRODUCTION}

Broadcast is a common communication method in a wireless environment. When base station wants to send information to many number of sensor nodes, broadcasting is a much more efficient method than send to each node individually. Broadcast authentication is a basic and important security mechanism in a WSN.

A wireless sensor networks may contain one or more base stations and hundreds or thousands of sensor nodes. Let us assume that each broadcast message originates from the base station. Compared to a base station, a sensor node is very limited in resources. A sensor node can broadcast messages by first unicasting the message to the base station, which then broadcasts the messages on the sensor nodes. The messages transmitted in a sensor network may reach the destination directly or may be forwarded by some intermediate nodes. To achieve authenticated broadcasts, it is necessary to establish an asymmetric mechanism in which only the sender can sign messages, and the receivers can only verify messages.

A number of broadcast authentication schemes have been proposed, especially for internet environment. Gennaro et al proposed a onetime signature scheme for stream authentication [1]. In this paper describes about a new efficient paradigm for signing digital streams. The problem is to prove the authentication is substantially different from the signing of regular messages. For this they proposed two solutions, the first one is finite stream which is entirely known to the sender and the second one is potentially infinite stream which not known to the sender in advance. When used in sensor networks this scheme not tolerates packet loss and one time public key scheme quite large.

One approach to reduce the size of the public key based signature signing and verification is to sign a number of packets together. The common property of this amortized approach [2] is need for buffering. In the authentication of digital streams, sender knows the whole packet sequence and buffering is responsible. However, in sensor networks broadcasting messages are usually unpredictable and time sensitive. So the sender and receiver buffering is problem in broadcast authentication.

Another approach is message authentication codes (MAC) [4]. MAC is an efficient symmetric cryptographic primitive for two-party authentication. In this sender and receiver shares the secrete keys, sender append a MAC to each message. This is not suitable for sensor networks because the master key will be compromised if one sensor is compromised. However, MAC is not suitable for broadcast communication without additional modification. Because the sender and its receivers share the same secret key, any one of the receivers can impersonate the sender and forge messages to other receivers. That is, both sender and receivers can sign messages. This problem stems from the symmetric property of MAC.

The improved MAC is described [4, 8] as instead of using one MAC key, the key pool is suggested to improve the robustness against spoofing. In this the communication overhead is occur because of the packet size is as large as the key pool size.

The Perrig proposed BIBA [5] which provides the very fast signature verification to achieve both efficient and resilient to packet loss in broadcast authentication. The BIBA is very efficient, but the key size is very large and so is the communication overhead. 
Perrig proposed $\mu$-TESLA [5, 6, and 7] based on one way hash chain function provides resilient to packet loss and has a low communication overhead, but receivers cannot verify the signature. In Perrig [5, 6, and 7] $\mu$-TESLA was extended to an authentication mechanism by replacing receiver buffering with sender buffering. As we discussed earlier, the sender buffering is not suitable for real time broadcast authentication and which is quite efficient and robust to packet loss.

Public key cryptography (PKC) is desirable for the broadcast authentication. The recent research has shed light on the public key schemes [10 and11]. The PKC are widely considered as impractical for sensor networks because of the computation and large size signature. In traditional only one key is used for whole life time so the message is compromised.

To make the public key system secure the RSA proposed [3] the 1024 bits parameters for broadcast authentication. The cost of using the traditional public key scheme in networks is high in both computation and communication. To avoiding this RSA uses separate public and private keys for encryption and decryption. The fundamental operation underlying RSA is modular exponentiation in integer rings and its security stems from the difficulty of factoring large integers. This is high cost due to length and requires more energy to transmit the signature.

To reduce the cost, many number of short length ECC keys are used. Compared to RSA, the prevalent public-key scheme of the Internet today, Elliptic Curve Cryptography (ECC) offers smaller key sizes, faster computation, as well as memory, energy and bandwidth savings and is thus better suited for small devices. While ECC operates on groups of points over elliptic curves and derives its security from the hardness of the elliptic curve discrete logarithm problem (ECDLP). While sub-exponential algorithms can solve the integer factorization problem, only exponential algorithms are known for the ECDLP. This allows ECC to achieve the same level of security with smaller key sizes and higher computational efficiency; ECC-160 provides comparable security to RSA-1024 and ECC-224 provides comparable security to RSA-2048.

In ShortPK, an efficient Short-term Public Key broadcast authentication scheme. In this paper describes [12] to use short-length public/private keys. This will reduce the security strength of public keys; so, instead of the traditional approach that uses one long key that is hard to break for a long period of time measured by years, they limit the lifetime of short public keys to a small period of time that is measured by minutes. The lifetime of each short public key is named as term. The lifetime of a sensor network is divided into a number of such terms, and assign a different public/private key pair for each term. A public key is only valid within its own lifetime, so broadcast authentication is still safe even if a public/private key pair is broken after its term, because any signature generated using that pair will be invalid. For instance, based on the table, it will take 233 machines to work together for about 24 hours to break an 80-bit ECC key. Therefore, if the lifetime of an 80-bit key is set to 5 minutes, to break it within this lifetime, adversaries need at least 66,000 computers to work simultaneously.

In ShortPK approach, broadcast authentication is less expensive, but problem is distributing these public keys to sensors because of the memory limitation, we cannot preload all the public keys into sensor's memory prior to deployment; even if we can, they must be kept secret until their corresponding terms.

Therefore, the public keys need to be broadcasted by the base stations. The broadcasted public keys must also be authenticated. The original message broadcast authentication problem is reduced to public key distribution problem. The progressive public key distribution scheme (PPKD) is used to achieve secure, efficient, and packet loss resilient authentication. In PPKD the total life time is divided into terms. Each term have separate keys. All the sensors maintain the same time interval. Some of the packets may not reach the sensor node in same time interval due to propagation delay. The sensors cannot decrypt the message in these situations.

A group key is a common public key that is shared between groups of users. Typically, group keys are distributed by sending them to individual users, either physically, or encrypted individually for each user using either that user's pre-distributed private key. A common use of group keys is to allow a group of users to decrypt a broadcast message that is intended for that entire group of users, and no-one else.

Let there be a trusted server which is given membership information to exercise group access control. When a client wants to join the group, the client and server mutually authenticate using an authentication protocol. Having been authenticated and accepted into the group, each member shares with the server a key, is called member's individual key. For group communications, the server distributes to each member a group key to be shared by all members of the group [18]. Consequently, the group key should be changed frequently. To achieve a high level of security, the group key should be changed after every join and leave so that a former group member has no access to current communications and a new member has no access to previous communications.

The Group Key Management Protocol (GKMP) [16], [17] which provides the ability to create and distribute keys within arbitrary-sized groups without the intervention of a global/centralized key manager. The GKMP combines techniques developed for creation of pair wise keys with techniques used to distribute keys from a KDC (i.e., symmetric encryption of keys) to distribute symmetric key to a group of hosts.

We proposed a new technique Tree based Short term Group key Algorithm (TSGA) to improve packet delivery ratio and reduce the packet loss due to failure in decryption.

\section{PROPOSED WORK}

The proposed new broadcast authentication scheme contains five phases such as key generation, depth creation, parent node selection, group key establishment and key distribution. During sensor node deployment all are preloaded with initial short term 80 bit ECC private keys. In key generation, all the sensor nodes generates $\mathrm{N}$ number of public keys depends on the key duration and phase duration. To form a group we divide the area into different depths based on the distance from base station node to sensor node. Lower depth nodes are selected as parent by the upper depth child nodes. In each key duration child nodes send their public key to parent node. Parent node computes the group key (group_key) and stored as parent_key then distribute to its child nodes. While forwarding broadcast message parent node encrypts the message using its parent_key, child nodes decrypts the message using group _key. 


\subsection{Key Generation}

A key pool consists of private and public keys. The private keys are preloaded into all the sensor nodes and the public_key is derived from this private key. Each node contains public and private keys

$\mathrm{Nk}=$ Number of public_keys for particular phase.

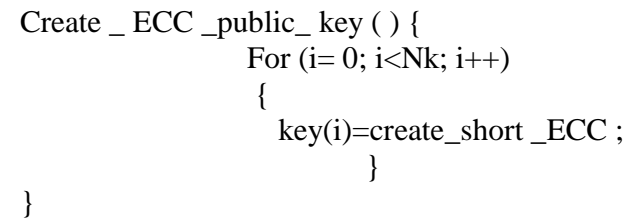

\section{Fig 1: Key pool generation}

During sensor node deployment all are preloaded with initial short term 80 bit ECC private keys. In key generation, all the sensor nodes generates $\mathrm{N}$ number of public keys depends on the key duration and phase duration

\subsection{Depth Creation}

The sink node creates the broadcast announcement message. The broadcast announcement message contains location of the sink node and depth of the corresponding sink node. The broadcast message encrypted with preloaded public_key. Already $\mathrm{N}$ number of public keys are loaded in all sensor nodes when it is deployed.

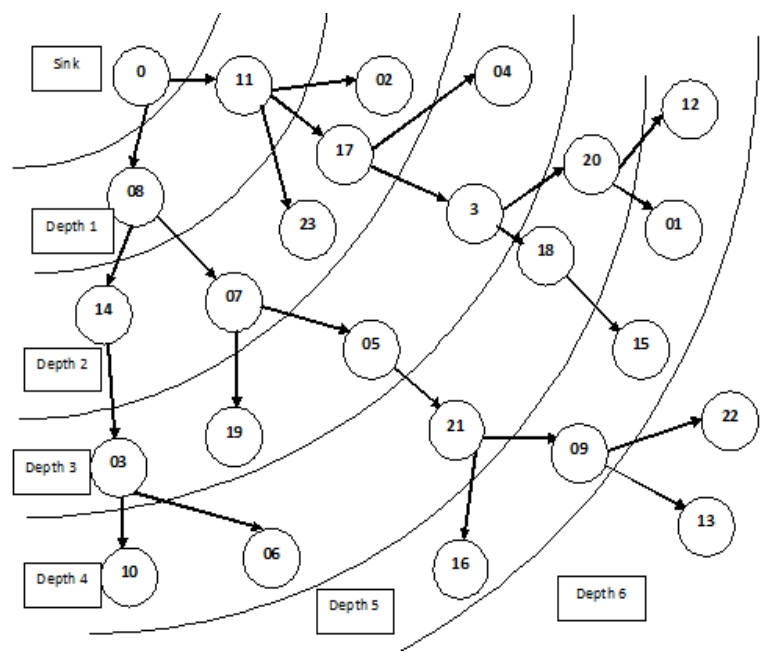

Fig 2: TSGA depth creation

dist - distance between the Sink to mobile node. $\mathrm{dl}$ - distance between the adjacent depth .

$$
\begin{gathered}
\text { Assign_depth (dist) }\{ \\
\text { If }(0<\text { dist }<\mathrm{dl}) \\
\text { Depth }=1 \\
\text { If }(2 * \mathrm{dl}<<\text { dist }<(2+1) \mathrm{dl}) \\
\text { Depth }=2 \\
\text { If }(\mathrm{i} * \mathrm{dl}<\mathrm{dist}<(\mathrm{i}+1) \mathrm{dl}) \\
\text { Depth }=\mathrm{i}
\end{gathered}
$$

Fig 3: Depth assignment
The sink node sends the broadcast announcement message to all the sensor nodes. Each node receives the broadcast message and decrypts the message. While decrypting the broadcast message receiver nodes find the distance between nodes to sink using location of the sink node. Based on the node distance it assigns depth. Each and every node notes down the depth and forwards the packets to all other nodes in network with their location and depth. In this every node should knows their depth and location.

It is based on the coverage area. If the $\mathrm{dl}$ value is reduced then the number of levels is increased.

\subsection{Parent Node Selection}

Receiving sensor nodes creates the one hop tree join message. The tree join message contains depth and node id. Then receiving sensor nodes broadcast the tree join message to the neighbour nodes. The Lower depth node sends tree reply message for the tree join message (higher depth nodes). Each node assigns parent node by receiving tree reply message. If sensor nodes receive multiple tree reply message from neighbour nodes it select short distance node as a parent node.

\subsection{Group key Establishment}

In this one parent node and their child nodes in the next depth forms the group. The group key is formed by their child keys in their group. Each child node sends the public_key to their parent node. The Parent node stores the child_keys and compute the group_key based on their child_keys using the group key for $\mathrm{i}$ th duration formula in figure 4. Parent node sends the group_key to their child nodes with short term duration.

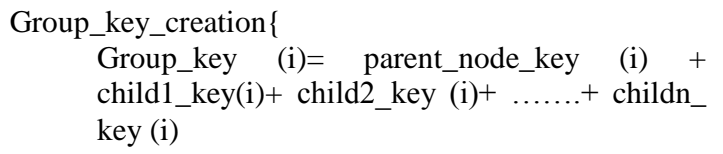

Fig 4: Group key for i th duration

\subsection{Key Distribution}

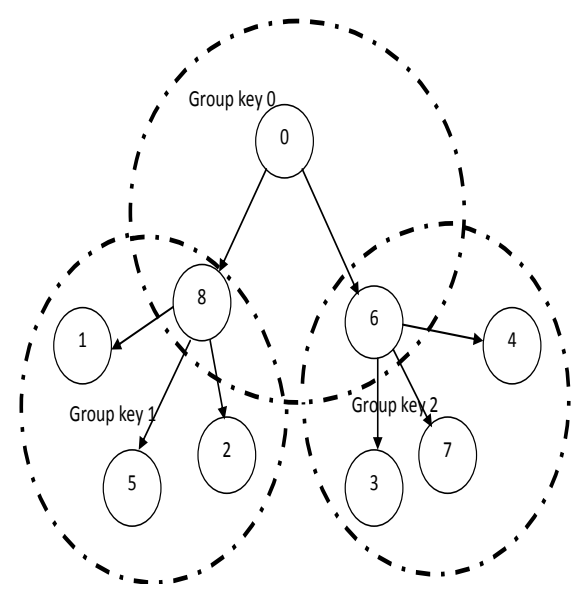

Fig 5: Group key establishment

We divide the total life time of the sensor networks into short term duration which is called phase and use Phi to represent the i-th phase. The parent node broadcasts the keys with 
message for phase Phi+1 during phase Phi itself. All the nodes have key pools of public and private keys. Each sensor nodes randomly select their public keys for next phase $\mathrm{P} h \mathrm{i}+1$ during the current phase Phi. In this every phase the key will changed automatically.

During phase $\mathrm{P} h \mathrm{i}$ the child nodes in a particular group send their child keys for next phase to the parent node. The parent node generates group key for next phase. Then parent node broadcast the group key for next phase to their child nodes in

Step 1: All the sensor nodes are preloaded with ECC short length private key.

Step 2: Create announcement message

$>$ Announcement message contains location, sink depth.

Encrypt the announcement message with the preloaded public key.

Step 3: The sink node broadcast the announcement message to all the sensor nodes.

Step 4: Receiving sensor nodes

$>$ Decrypt the announcement message by its private key.

$>$ Calculate the distance from node to sink.

Step 5: Compute depth and forwarded to other nodes.

Step 6: Receiving sensor nodes broadcast the one hop tree join message with depth and node id.

Step 7: Lower depth node sends tree reply message for the tree join message.

Step 8: Each node assigns parent node by receiving tree reply message.

Step 9: If multiple tree reply message received by the node, select short distance as a parent node.

Step 10: One parent node and their child nodes in the next depth forms the group.

Step 11: Each child node sends the public key to their parent node.

Step 12: Parent node stores the child keys and compute the group key based on their child keys.

Step 13: Parent node sends the group key to their child nodes with term duration.

Step 14: Broadcast message encrypted by parent node by parent key.

Step 15: Child nodes decrypt the message by using their group key.

Fig 6: TSGA Proposed Algorithm their group during the current phase. By dynamically changing the group keys in predefined short duration and depth creation to avoid the problems topology changes.

All the sensor nodes divided the total life time into short term duration. The parent node encrypts the broadcast message by parent key and broadcast throughout the group.

Child nodes receive and decrypt the message by using this group key. Then child node in the current depth will act as a parent node for their next depth nodes. In this the decrypted message is again encrypted by their parent node.

The packet loss due to propagation delay will be reduced. Because of group keys used for only that particular group instead of the short keys used for whole network. In this tree based group key algorithm performs better when minimum key duration condition also. So the sensors easily decrypt the message in these situations.

\section{PERFORMANCE ANALYSIS}

We evaluate TSGA method performance through NS-2 [14] simulations. In particular we used NS-2.32 package as our tool to simulate the proposed protocol. NS-2 is an object oriented discrete time event simulator written in $\mathrm{C}++$, with an OTcl interpreter and its modular design made it to be extensible.

Simulation parameters are listed in Table.1. We created a 100 sensor nodes and all are randomly placed within a $1000 \mathrm{~m} \mathrm{x}$ $1000 \mathrm{~m}$ area. Sensor node coverage area is defined as $100 \mathrm{~m}$. Each simulation is executed for 200 seconds. The Constant Bit Rate (CBR) application used between randomly selected source and destination pair. The size of the data packet is 38 bytes and the transmission rate varied from $0.1 \mathrm{~s}$ to $0.01 \mathrm{~s}$.

Table 1. Simulation Parameters

\begin{tabular}{|l|l|l|}
\hline S. No & Parameter & Values \\
\hline 1 & Simulation area & $1000 \times 1000 \mathrm{~m}$ \\
\hline 2 & Node coverage area & $100 \mathrm{~m}$ \\
\hline 3 & Number of nodes & 100 \\
\hline 4 & Simulation time & $200 \mathrm{~s}$ \\
\hline 5 & Message interval time & $0.02 \mathrm{~s}$ \\
\hline 6 & Average packet size & $38 \mathrm{Bytes}$ \\
\hline 7 & Key length & $80 \mathrm{bits}$ \\
\hline 8 & Key duration & $4-30 \mathrm{sec}$ \\
\hline 9 & Node Energy & $100 \mathrm{~J}$ \\
\hline
\end{tabular}

Packet end to end delay is calculated by subtracting the time at which packet is received by the destination from the time it was delivered from the source.

In our protocol, if the key duration increases, the number of group key generated per phase is decreased and the packet delivery ratio will be increased. Secondly, the key distribution involves with in a depth so that key distribution delay and packet loss will be reduced. 


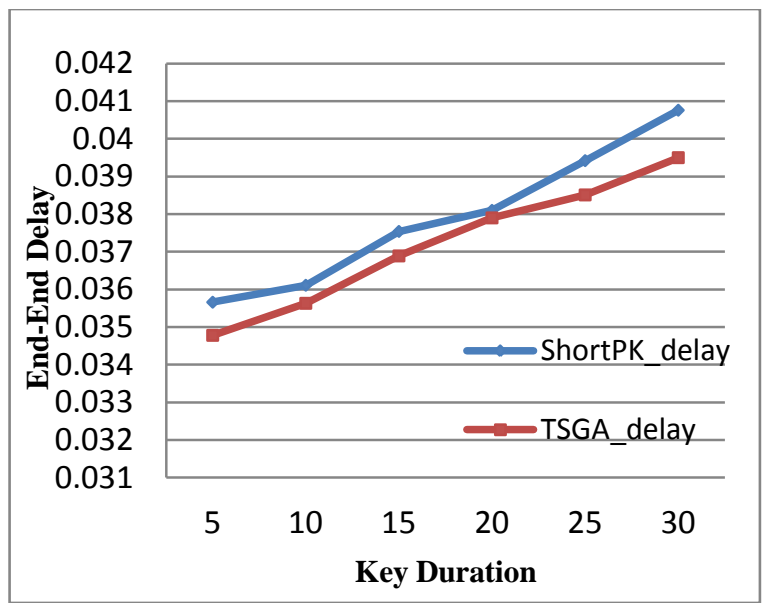

Fig 7: Key Duration Vs End-End delay

In our proposed algorithm the group keys are changed after particular time duration. The term duration is varied with respect to the applied data traffic. We performed the simulation by varying the key duration. While varying the key duration parameter all other parameters are kept constant. Key duration varied from 5-30 sec and data packet interval is fixed as 0.02 seconds. In our scheme end to end delay is smaller than shortPK approach.In Fig. 7 the algorithm shows, end to end delay is less when compared with ShortPK.

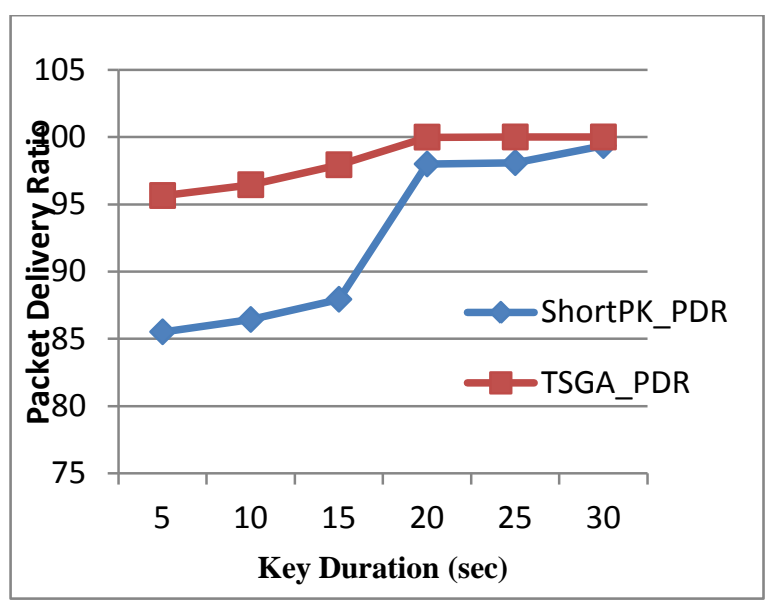

Fig 8: Key Duration Vs Packet Delivery Ratio

We performed the simulation by varying the key duration. While varying the key duration parameter all other parameters are kept constant. Key duration varied from 5-30 sec and data packet interval is fixed as 0.02 seconds.

Generally if the key duration increases, the number of group key generated per phase is decreased and the packet delivery ratio will be increased. Secondly, the key distribution involves with in a depth so that key distribution delay and packet loss will be reduced. Fig.7 and Fig.8 shows our protocol performed well in terms of packet delivery ratio for each duration and average end-to-end delay.

We performed the simulation by varying the number of nodes. While varying the number of nodes parameter all other parameters are kept constant.

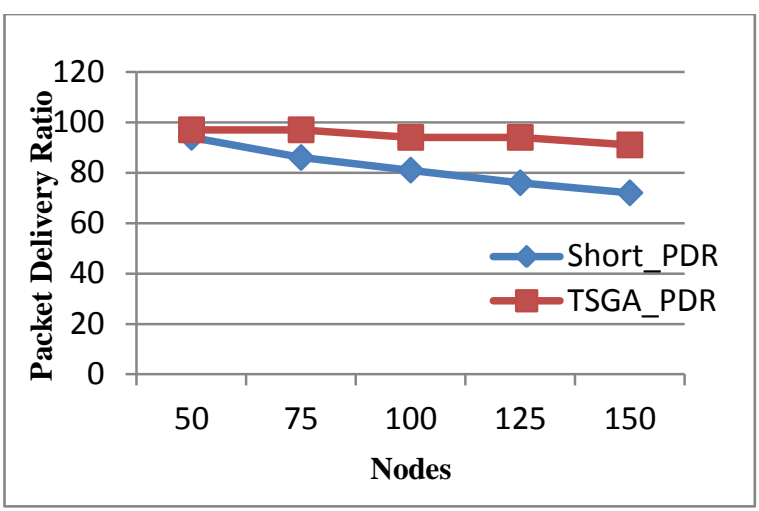

Fig 9: Nodes Vs Packet Delivery Ratio

Number of nodes varied from 50-150 nodes and data packet interval is fixed as 0.02 seconds. In Fig. 9 shows TSGA model the keys are different for all the groups in a network. So the packet loss due to propagation delay is reduced.

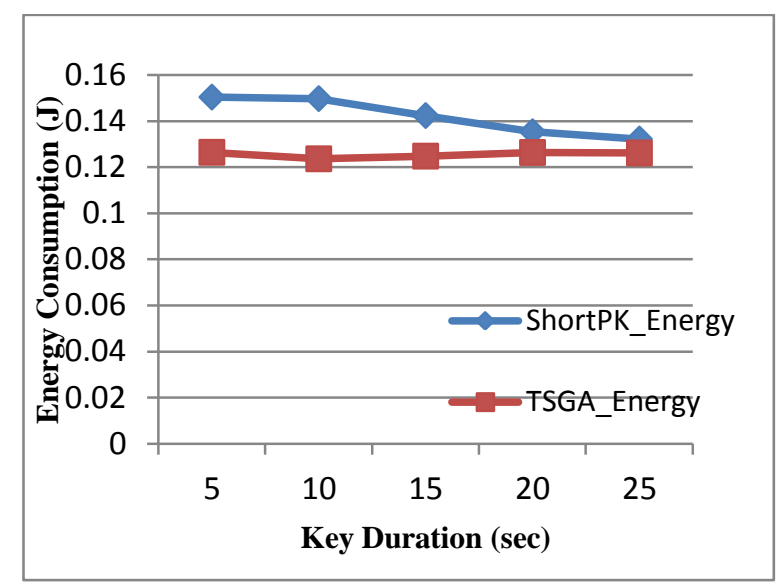

Fig 10: Key Duration Vs Energy Consumption

In our protocol, Fig. 10 shows, if the key duration increases, the number of group key generated per phase is decreased and the consumed energy will be decreased. Secondly, the key distribution involves with in a depth so that key distribution delay and consumed energy will be reduced.

\section{CONCLUSIONS}

Comparing our proposed scheme with shortPK [12], an efficient broadcast authentication protocol for WSNs. our proposed scheme is performed than shortPK in terms of message delivery and End to End delay with lower energy consumption. Our protocol achieves high security for broadcast message by dynamically changing the group keys in predefined short duration. In future work we plan to implement hierarchical hash function to further enhance the security in our TSGA method.

\section{REFERENCES}

[1] Gennaro. R. and Rohatgi. P., 1997. "How to sign digital streams", In Technical report, IBM T.J.Watson Research Centre.

[2] Lam. S. and Wong. C., 1998. "Digital signatures for flows and multicasts", In Proceedings of the 6th IEEE International Conference on Network Protocols (ICNP '98) Austin, TX. 
[3] Boneh. D. and Durfee. G., 1999. "Cryptanalysis of RSA with private key d less than N0.292", Lecture Notes in Computer Science 1592, 1-9.

[4] Golle. P. and Modadugu. N., 2001. "Authenticating streamed data in the presence of random packet loss". In Proceedings of the Network and Distributed System Security Symposium (NDSS ’01), San Diego, CA.

[5] Perrig. A., 2001. "The $\mathrm{BiBa}$ one-time signature and broadcast authentication protocol", In Proceedings of the ACM Conference on Computer and Communications Security (CCS ’01). Philadelphia PA, USA, 28-37.

[6] Canetti. R., Perrig. A., Song. D. and Tygar. D., 2001. "Efficient and secure source authentication for Multicast", In Proceedings of Network and Distributed System Security Symposium (NDSS ’01). San Diego.

[7] Cullar. D. Perrig. A., Szewczyk. R., Tygar. J.D and Wen V., 2001. "Spins: Security protocols for sensor networks", In Proceedings of the 7th Annual International Conference on Mobile Computing and Networking (MobiCom '01). Rome, Italy, 189-199.

[8] Song. D., Tygar. J.D. and Zuckerman. D., 2002. "Expander graphs for digital stream authentication and robust overlay networks", In Proceedings of IEEE Symposium of Security and Privacy (IEEE S\&P '02). Oakland.

[9] Donggang Liu and Peng Ning, 2002. "Efficient Distribution of Key Chain Commitments for Broadcast Authentication in Distributed Sensor Networks", Department of Computer Science, North Carolina State University, available at dliu@unity.ncsu.edu, ning@csc.ncsu.edu.

[10] Eberle. H., Gura. N., Patel. A., Shantz. S. C. and Wander. A., 2004. "Comparing Elliptic Curve Cryptography and RSA On 8-Bit CPUs". In Proceedings of the Workshop on Cryptographic Hardware and Embedded Systems (CHES '04). Cambridge, MA, USA.

[11] Ning. P., 2006. "Tinyecc: Elliptic Curve Cryptography for Sensor Networks", Cyber Defense Lab, North Carolina State University, available at http://discovery.csc.ncsu.edu/pning/software/TinyECC/in dex.html.

[12] Peng Ning, Ronghua Wang, Wenliang Du and Xiaogang Liu, 2010. "shortPK: A Short-Term Public Key Scheme for Broadcast Authentication in Sensor Networks", North Carolina State University, ACM Transactions on Sensor Networks.
[13] Colegrove. A., Harney. H. and McDaniel. P., 2001. "Principles of policy in secure groups". In network and distributed systems security symposium.

[14] Network Simulator 2, available at http://www.isi.edu/nsnam/ns/.

[15] Cristina Nita-Rotaru, Gene Tsudik Yair Amir and Yongdae Kim, 2003. "On the Performance of Group Key Agreement Protocols", University of California, ACM Transactions on Sensor Networks.

[16] Fenner, W, 1997. "Internet group management protocol", version 2, RFC-2236.

[17] Harney, H., and Muckenhirn, C, 1997. "Group key management protocol (gkmp) architecture", IETF Request for Comments, RFC 2094.

[18] Ravi Krishna.B and Vijaya saradhi M.V., 2009. "A Group Key Management Approach for Multicast Cryptosystems", journal of Theoretical and Applied Information Technology, 104-109.

\section{AUTHORS PROFILE}

M. Premkumar is an assistant professor at the Department of Electronics and Communication Engineering, Sri Subramaya College of Engineering and technology - Palani,Tamilnadu. $\mathrm{He}$ received the Master degree from Karpagam college of Engineering, Coimbatore and the Bachelor degree from Sri Subramaya College of Engineering and technology, Palani, in 2012 and 2009. His research interests include authentication in ad hoc and sensor networks, and security in peer to peer systems.

M. Kathiravan is an assistant professor at the Department of Electronics and Communication Engineering, Dr. Nallini Institute of Engineering and Technology-Dharapuram, Tamil Nadu. He received the Master degree (2012) and the Bachelor degree (2008) from Karpagam college of Engineering, Coimbatore. His research interests include specification, verification and testing of communication protocols; network security and distributed systems

R.Thhirukkumaran received the Master degree from Karpagam college of Engineering, Coimbatore and the Bachelor degree from Kongu College of Engineering, Erode, in 2012 and 1995. His research interest also includes the field of multimedia wireless networks: convergence, DSP, QoS and security. 